confusion. Take, for example, an item specifically identified by Cheng: life satisfaction. Successful aging was originally posited as inextricably linked to life satisfaction; however, subsequently, life satisfaction has been identified as a predictor (Roos and Havens, 1991), indicator (Leonard, 1981), and component (Pruchno et al., 2010) of successful aging. Further, several qualitative studies have been conducted with laypersons specifically differentiating between successful aging and life satisfaction, e.g. Fisher (1995). As Cheng illuminates and these examples highlight, the language of successful aging is rife with conceptual confusion - an inhibitive force in the advancement of the field - that must be addressed.

While it may be unrealistic to expect the development of a single, universally accepted operationalization of successful ageing, it is important to acknowledge the diversity of successful aging conceptualizations, e.g. from culturally and geographically diverse sources, and to look toward the practical utility of successful aging models. In order for models of successful aging to demonstrate real world applicability, it is imperative that these theoretical constructs are investigated in practical settings, e.g. longitudinal population-based cohort studies, and their associations with public health outcomes, e.g. health service utilization, are examined. The successful aging paradigm will be greatly strengthened through the application of wellinformed conceptualizations of successful aging that have been validated in real-world contexts.

doi:10.1017/\$1041610214001288

\section{Antipsychotics for behavioral and psychiatric symptoms of dementia: a reaudit in a specialist inpatient service}

The use of antipsychotics for the management of

behavioral and psychiatric symptoms of dementia (BPSD) remains highly controversial. These drugs are well known to be associated with increased mortality from cerebrovascular events, as well as with falls, cognitive impairment, and other serious side effects. In 2009, a UK target was set to reduce their use in dementia patients by two-thirds over a three-year period (Banerjee, 2009).

In an earlier publication, we reported on the prescribing of antipsychotics to a group of dementia patients in a specialist inpatient service carried out in 2007 (Haw et al., 2008). The audit was conducted at St Andrew's, Northampton, UK, a charitable foundation which acts as a tertiary referral center. Within the hospital, there is an

\section{Conflict of interest}

None.

\section{References}

Cosco, T. D., Prina, A. M., Perales, J., Stephan, B. C. and Brayne, C. (2014). Operational definitions of successful aging: a systematic review. International Psychogeriatrics, 26, 373-381.

Fisher, B. J. (1995). Successful aging, life satisfaction, and generativity in later life. International fournal Aging and Human Development, 41, 239-250.

Leonard, W. M., 2nd (1981). Successful aging: an elaboration of social and psychological factors. International Fournal Aging and Human Development, 14, 223232.

Pruchno, R. A., Wilson-Genderson, M. and Cartwright, F. (2010). A two-factor model of successful aging. Fournals of Gerontology: Series B Psychological Sciences and Social Sciences, 65, 671-679.

Roos, N. P. and Havens, B. (1991). Predictors of successful aging: a twelve-year study of Manitoba elderly. American Fournal of Public Health, 81, 63-68.

\section{THEOdore D Cosco \\ Cambridge Institute of Public Health, Department of Public Health and Primary Care, University of Cambridge, Cambridge, UK \\ Email: tdc33@cam.ac.uk}

inpatient service for older adults with dementia and challenging behavior and those requiring secure psychiatric care. There is also a unit for patients with Huntington's disease. Although prescribing standards (derived from the National Institute for Health and Clinical Excellence (NICE) dementia guideline (National Collaborating Centre for Mental Health, 2007) and Royal College of Psychiatrists prescribing update (Royal College of Psychiatrists, 2005) were generally satisfactory, some aspects showed room for improvement. We therefore decided to repeat the audit to see if antipsychotic usage for managing BPSD had declined and to see if prescribing standards had improved compared with 2007.

The reaudit was carried out in June and July 2012 and once again involved a cross-sectional survey of inpatients with BPSD on four wards for older adults and one ward for patients with Huntington's disease. We scrutinized medication charts and case notes and questioned the treating psychiatrist using a three-page, 40-item structured proforma taking approximately $45 \mathrm{~min}$ to complete per patient, 
which is available from the authors on request. The survey was considered to be an audit and therefore Research Ethics Approval was not deemed necessary. It was approved by the hospital's head for Clinical Effectiveness.

In the 2012 audit there were 37 patients with BPSD, of whom $28(76 \%)$ were prescribed antipsychotics for this (the 2007 figure was $56 \%$ ). The median total percentage dose of antipsychotic had increased from $27 \%$ in 2007 to $38 \%$ in 2012. BPSD was rated by the treating clinician as severe in a lower proportion of cases $(89 \%$ in 2007 vs. $68 \%$ in 2012 ). Once again the most commonly prescribed antipsychotics were secondgeneration drugs (olanzapine $50 \%$ in 2007 vs. $39 \%$ in 2012$)$; quetiapine ( $19 \%$ in 2007 vs. $25 \%$ in 2012$)$; risperidone $(23 \%$ in 2007 vs. $21 \%$ in 2012), and the main symptoms targeted were, as before, aggression followed by agitation, psychosis, and sexual disinhibition. A higher proportion of patients were already receiving an antipsychotic when transferred to our service $(79 \%$ in 2007 vs. $89 \%$ in 2012). Encouragingly, in 2012, slightly greater use had been made of non-pharmacological strategies to try and manage BPSD (75\% in 2007 vs. $79 \%$ in 2012), while clinicians reported much greater use of other pharmacological strategies, for example, memantine and acetylcholinesterase inhibitors ( $18 \%$ in 2007 vs. $100 \%$ in 2012). In 2012, $24 \%$ of patients had been tried on memantine, $19 \%$ on acetylcholinesterase inhibitors, and $9 \%$ on both. Also of note, a higher proportion of relatives had been involved in the decision to prescribe an antipsychotic (60\% in 2007 vs. $100 \%$ in 2012), while the antipsychotic was started at a lower dose and was titrated more slowly (50\% in 2007 vs. $100 \%$ in 2012), and withdrawal of the antipsychotic had been attempted in a slightly greater proportion of patients ( $47 \%$ in 2007 vs. $54 \%$ in 2012 ). However, in 2012 there was greater use of antipsychotic polypharmacy (4\% in 2007 vs. $11 \%$ in 2012) and for no patient had the off-label use of the antipsychotic been documented in the case notes by the consultant psychiatrist. In 2012, of the three patients receiving antipsychotic polypharmacy, one was prescribed haloperidol and olanzapine, the second zuclopenthixol and quetiapine, and the third was prescribed haloperidol and zuclopenthixol decanoate depot injection.

We were somewhat surprised at the greater use of antipsychotics for BPSD in the reaudit. It must be emphasized that almost all the patients concerned had been transferred to our tertiary referral service from other psychiatric hospitals because of difficulty in managing their aggressive and/or challenging behavior. We think the increased use of antipsychotics in our specialist inpatient service is likely to reflect our clinical impression that a greater proportion of dementia patients now being referred to our service are presenting with aggressive and challenging behavior. A higher percentage are now detained and already receiving an antipsychotic at the time of their admission. The audit standards showed a modest improvement in many areas compared with the earlier audit but there was again room for improvement, particularly in case note documentation. Given the justifiable pressures to reduce the use of antipsychotics for managing BPSD, we feel it is essential that we continue to closely monitor and audit our prescribing practice and would be interested to hear about the management of BPSD and in particular rates of antipsychotic prescribing for BPSD in other tertiary referral centers worldwide.

\section{Conflict of interest}

None.

\section{References}

Banerjee, S. (2009). The use of antipsychotic medication for people with dementia: time for action. Available at http://s395229360.onlinehome.us/Research/Digest/NLPs/ BanerjeeReportOnGeriatricNeurolepticUse.pdf; last accessed 6 September 2013.

Haw, C., Stubbs, J. and Yorston, G. (2008). Antipsychotics for BPSD: an audit of prescribing practice in a specialist psychiatric inpatient unit. International Psychogeriatrics, 20, 790-799.

National Collaborating Centre for Mental Health. (2007). Dementia: A NICE-SCIE Guideline on Supporting People with Dementia and Their Carers in Health and Social Care. National Clinical Practice Guideline, No. 42. Leicester, UK: British Psychological Society. Available at: http://www.guidance.nice.org.uk/cg42; last accessed 6 September 2013.

Royal College of Psychiatrists. (2005). Atypical Antipsychotics and Behavioural and Psychiatric Symptoms of Dementia. Guidance note of The Royal College of Psychiatrists Faculty for the Psychiatry of Old Age. Available at: http://www.rcpsych.ac.uk/PDF/BPSD.pdf; last accessed 6 September 2013.

CAMIlla Haw, ${ }^{1,2}$ MARTINE STOFFEls, ${ }^{3}$
DEBASIAH DAS PURKAYASTHA ${ }^{3}$
AND SOFI SUDAD ${ }^{3}$
${ }^{1}$ St Andrew's Academic Centre, St Andrew's,
Cliftonville, Northampton NN1 5DG, UK
Email: chaw@standrew.co.uk
${ }^{2}$ School of Health, Northampton University,
Northampton, UK
${ }^{3}$ St Andrew's Healthcare, Northampton, UK

